\title{
A man with cardiac Lyme borreliosis
}

\author{
Jarne M. van Hattem MD, Jan T. Keijer MD PhD, Robin Nijveldt MD PhD
}

$\mathrm{A}$ 36-year-old man who had been previously healthy was admitted to the coronary care unit after an episode of syncope. Two weeks previously, he had experienced transient pain and stiffness in his left upper leg, which resolved within two days. A week later he had dizziness when standing up, palpitations and shortness of breath. This was followed by pain in the large joints of his left arm in the days preceding his hospital admission. He was a nonsmoker, used alcohol only occasionally and did not use any medication or drugs. There was no history of recent travel, and he could not recall any insect bites or skin lesions.

On examination, his blood pressure, body temperature and oxygen saturation were normal, but he had an irregular heart rate of 48 beats/ min. Auscultation of the heart and lungs was normal. There were slightly enlarged lymph nodes palpable in the left groin, and the left elbow and shoulder were painful to palpation. No swelling or redness of any of the joints or skin was visible. Electrocardiography showed temporary total atrioventricular block (not shown). Later that day, second-degree Mobitz type I atrioventricular block with intermittent junctional escape developed (Appendix 1, available at www.cmaj.ca/lookup/suppl/doi:10.1503/ cmaj.141468/-/DC1).

The explanation for our patient's presentation was not immediately clear. Our initial differential diagnosis for his presentation is found in Box 1 . Based on his younger age (because risk of idiopathic fibrosis tends to increase in older people), lack of medication use and unremarkable medical history (e.g., no history of aortic valve surgery, Steinert disease or amyloidosis), we expanded our diagnostic workup. Investigations for myocardial infarction and calcification of the aortic valve annulus were negative (i.e., no elevation in cardiac enzymes, and normal chest radiography and transthoracic echocardiography).

Because of our patient's young age, the absence of other risk factors and his history of arthralgia, we requested a serum enzyme-linked immunosorbent assay (ELISA) analysis for Lyme disease on the day of admission. The next day, the assay showed elevated Borrelia immunoglobulin $\mathrm{G}(\operatorname{IgG})(498 \mathrm{U} / \mathrm{mL})$ and immunoglobulin $\mathrm{M}(\mathrm{IgM})(2.7 \mathrm{U} / \mathrm{mL})$ levels, and a positive IgG and IgM Borrelia immunoblot, confirming the diagnosis of Lyme disease. We started treatment with intravenous ceftriaxone $2000 \mathrm{mg}$ daily for seven days.

Cardiovascular magnetic resonance imaging (MRI) was performed five days after admission for further cardiac evaluation. Dynamic (cine) cardiovascular MRI showed normal systolic ventricular function without any wall motion abnormalities. $T_{2}$-weighted imaging did not show evidence of acute inflammation; however, the late gadoliniumenhanced images showed hyperenhancement of the epicardial midlateral wall (Figure 1), consistent with myocarditis, which was presumably too subtle to detect with $T_{2}$-weighted imaging. These findings on cardiovascular MRI in combination with positive Borrelia IgM antibodies were strongly suggestive of Lyme carditis.

The patient's condition gradually improved, with resolution of atrioventricular block and PR interval shortening to a normal length of $180 \mathrm{~ms}$ after five days (Appendix 2, available at www. cmaj.ca/lookup/suppl/doi:10.1503/cmaj.141468/ -/DC1). He was discharged home with oral treatment with doxycycline $100 \mathrm{mg}$ twice daily for two more weeks.

\section{KEY POINTS}

- Lyme carditis should be considered in younger patients with atrioventricular conduction disorders, even without a history of tick bite.

- The diagnosis of Lyme carditis is based on the combination of clinical presentation, signs of Lyme disease (e.g., tick bite, erythema migrans) and laboratory findings.

- Cardiovascular magnetic resonance imaging has become the primary tool for noninvasive assessment of myocardial inflammation in patients with suspected myocarditis and can be supportive for the diagnosis of Lyme carditis.

- When Lyme carditis is promptly recognized and treated with intravenous antibiotics, introduction of a permanent pacemaker can be avoided. 
The next week, he was seen in the outpatient clinic with complaints of an asymmetric facial expression, headache, photophobia, and pain of the lower back and right hip. Physical examination showed nuchal rigidity, unilateral facial nerve paralysis and a radicular syndrome of the right leg. A lumbar puncture showed monocytic pleocytosis, elevated protein content and an increase in cerebrospinal fluid IgG. ELISA showed an ele-
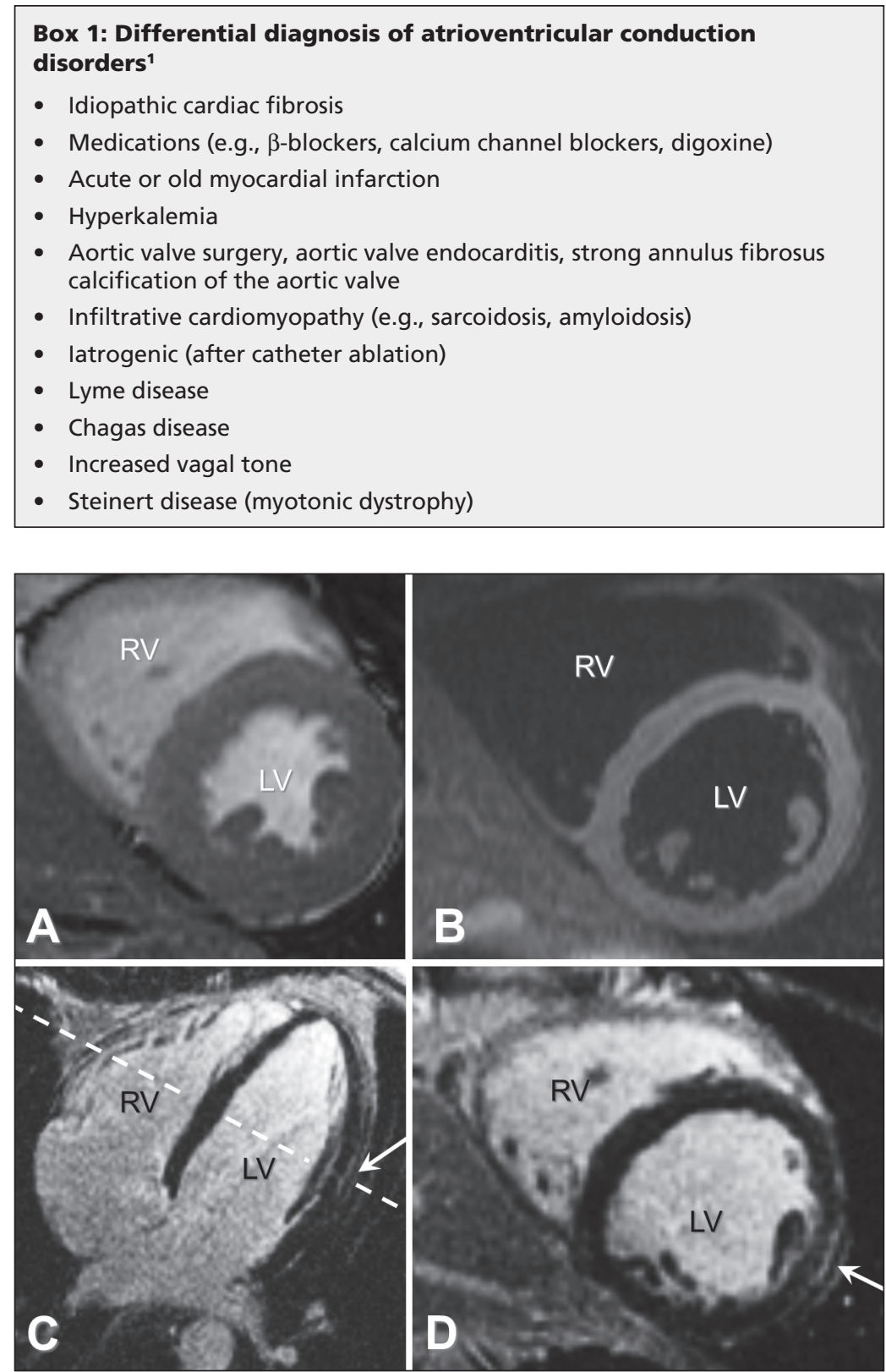

Figure 1: Cardiovascular magnetic resonance images showing normal systolic left ventricular function on cine imaging without wall motion abnormalities ( $A$, short-axis end-systolic phase). Panel $B$ shows a $T_{2}$-weighted image without high signal intensity in the myocardium, meaning that there is no evidence of acute myocardial inflammation. Panels $C$ and $D$ show late gadoliniumenhanced images, with a small area of epicardial hyperenhancement midanterolateral (white arrow) on the 4-chamber view (C) as well as on the short-axis view (D). Note: $L V=$ left ventricle, $R V=$ right ventricle. The dotted line indicates the section for images A, B and D. vated Borrelia IgG level $(333 \mathrm{U} / \mathrm{mL})$ and a normal Borrelia IgM level $(0.1 \mathrm{U} / \mathrm{mL})$; neuroborreliosis with meningopolyradiculitis, also known as Garin-Bujadoux-Bannwarth syndrome, was diagnosed. The electrocardiogram showed no abnormalities. Doxycycline was stopped and the patient was again given intravenous ceftriaxone $2000 \mathrm{mg}$ daily, for another two weeks. He recovered completely.

\section{Discussion}

Our patient presented with syncope from heart block, and after investigation, Lyme disease was diagnosed.

Lyme disease, also known as Lyme borreliosis, is a tick-borne infectious disease caused by spirochetes of the Borrelia burgdorferi sensu lato species. ${ }^{2}$ In North America, only B. burgdorferi sensu stricto has been isolated. ${ }^{3}$ In a prospective, population-based survey of cases of Lyme disease in southern Sweden, the disease was mostly caused by Borrelia afzelii. ${ }^{4}$ Less often it is caused by Borrelia garinii and B. burgdorferi. ${ }^{5}$ Borrelia spirochetes are transmitted to humans by bites from Ixodes ricinus complex ticks.

Presentations of Lyme disease tend to cluster along three lines: 1) an early localized stage with skin manifestations such as erythema migrans or Borrelia lymphocytoma, sometimes preceded by migratory arthralgia; 2) an early disseminated form, with multiple erythema migrans, neuroborreliosis, arthritis or carditis; and 3) late disease (which may involve the skin, joints, and central or peripheral nervous system). ${ }^{2}$

\section{Lyme carditis}

When Borrelia spirochetes infiltrate the cardiac tissue, the disease is called cardiac Lyme borreliosis or Lyme carditis. The spirochetes cause transmural inflammation, necrosis and fibrosis. An autoimmune reaction against myocardial tissue may also play a role in Lyme carditis.

Lyme carditis is a relatively rare finding in Lyme disease, but seems to be more prevalent in North America than in Europe. Myocarditis occurs in about $10 \%$ of adults in the United States who are not receiving treatment for Lyme disease, whereas in Europe this manifestation is only present in $0.4 \%-4 \%$ of patients with Lyme borreliosis. ${ }^{6}$ In a Swedish study, 7 of $1471(0.5 \%)$ patients with Borrelia infections presented primarily with Lyme carditis. ${ }^{3}$ The difference between incidence in North America and Europe may be explained by differences between causative Borrelia species.

The most common finding in Lyme carditis is 
an atrioventricular block related to dysfunction of the conduction system; in some cases, a temporary pacemaker may be needed. ${ }^{2} \mathrm{~A}$ less common finding is decreased cardiac contractility due to perimyocarditis. ${ }^{6}$

\section{Diagnosis}

The diagnosis of Lyme carditis is notoriously challenging, and is based on the combination of clinical presentation (arthralgia in combination with conduction disorders), signs of Lyme disease (tick bite, erythema migrans) and laboratory findings. ${ }^{3}$ Diagnostic tests include ELISA, immunofluorescence assay and Western blot. ${ }^{2,7}$ A seroconversion or a substantial rise in binding activity, intensity or number of reactive bands in ELISA or immunofluorescence assay in two consecutive sera establishes the diagnosis of Lyme borreliosis, but negative serology does not exclude it. ${ }^{7}$ Although conduction disturbances are rare, it is important to acquire an electrocardiogram in every case of suspected Lyme disease. ${ }^{10}$ Cardiovascular MRI has become the primary tool for noninvasive assessment of myocardial inflammation in patients with suspected myocarditis. ${ }^{8}$ Although not performed routinely, cardiovascular MRI can be supportive for the diagnosis of Lyme carditis.

\section{Treatment}

Treatment of Lyme carditis, according to our local protocol, was either oral doxycycline for 30 days, or 14 days of intravenous cephalosporins. ${ }^{6}$ This protocol is based on a study suggesting that oral doxycycline is as effective as ceftriaxone in early disseminated Lyme disease. ${ }^{9}$

As suggested by our case, however, it may not always be safe to switch from intravenous to oral antibiotics in Lyme carditis, which raises the question of whether all patients with Lyme carditis should receive intravenous antibiotics. A guideline from the International Lyme and Associated Diseases Society Working Group ${ }^{7}$ advises intravenous treatment upon failure of oral medications and as the first line of therapy for certain conditions including heart block, encephalitis, meningitis, optic neuritis and joint effusions. In an updated Dutch guideline,${ }^{10}$ treatment with ceftriaxone instead of doxycycline is advised for "severe cases of Lyme carditis," defined as a PR interval greater than $300 \mathrm{~ms}$, or a second- or third-degree atrioventricular block.

The total atrioventricular block usually disappears within a week and the other conduc- tion abnormalities within a period of six weeks. Temporary pacing is necessary in about a third of patients. A permanent pacemaker appears to be only rarely needed, but may be placed mistakenly if the diagnosis of Lyme carditis is missed. ${ }^{3,10}$

\section{References}

1. Mangrum JM, DiMarco JP. The evaluation and management of bradycardia. N Engl J Med 2000;342:703-9.

2. Stanek G, Wormser GP, Gray J, et al. Lyme borreliosis. Lancet 2012;379:461-73.

3. Steere AC. Lyme disease. N Engl J Med 2001;345:115-25.

4. Berglund J, Eitrem R, Ornstein K, et al. An epidemiologic study of Lyme disease in southern Sweden. N Engl J Med 1995;333: 1319-27.

5. Hengge UR, Tannapfel A, Tyring SK, et al. Lyme borreliosis. Lancet Infect Dis 2003;3:489-500.

6. Speelman P, de Jongh BM, Wolfs TF, et al. Guideline 'Lyme borreliosis' [article in Dutch]. Ned Tijdschr Geneeskd 2004; 148:659-63

7. Cameron D, Gaito A, Harris N, et al. Evidence-based guidelines for the management of Lyme disease. Expert Rev Anti Infect Ther 2004;2(Suppl):S1-13.

8. Friedrich MG, Sechtem U, Schulz-Menger J, et al. Cardiovascular magnetic resonance in myocarditis: a JACC white paper. J Am Coll Cardiol 2009;53:1475-87.

9. Dattwyler RJ, Luft BJ, Kunkel MJ, et al. Ceftriaxone compared with doxycycline for the treatment of acute disseminated Lyme disease. N Engl J Med 1997;337:289-94.

10. CBO (Dutch Quality Institute for Healthcare). Guideline Lymeborreliosis [article in Dutch]. Utrecht (the Netherlands); 2013. Available: www.diliguide.nl/document/1314/file/pdf (accessed 2014 Oct. 27)

Affiliations: Department of Medical Microbiology (van Hattem), Academic Medical Center; Department of Cardiology (Keijer), Tergooi Hospital, Hilversum; Department of Cardiology (Nijveldt), VU University Medical Center, Amsterdam, the Netherlands

Contributors: Jarne van Hattem drafted the manuscript, which Jan Keijer and Robin Nijveldt revised. All of the authors were involved in the patient's care during admission, approved the final manuscript and agreed to act as guarantors of the work.

Acknowledgment: The authors thank Paul Bouma for reviewing this manuscript.

\section{Resources for clinicians and patients}

- The Canadian Lyme Disease Foundation: http://canlyme.com

- Treatment guidelines by the International Lyme and Associated Diseases Society: www.ilads.org/lyme/treatment-guideline.php

- Clinical practice guidelines by the Infectious Diseases Society of America: http://cid.oxfordjournals.org/content/43/9/1089.full

- Information for health care workers and patients from the Public Health Agency of Canada: www.phac-aspc.gc.ca/id-mi/lyme-eng.php

The section Cases presents brief case reports that convey clear, practical lessons. Preference is given to common presentations of important rare conditions, and important unusual presentations of common problems. Articles start with a case presentation (500 words maximum), and a discussion of the underlying condition follows (1000 words maximum). Visual elements (e.g., tables of the differential diagnosis, clinical features or diagnostic approach) are encouraged. Consent from patients for publication of their story is a necessity. See information for authors at www.cmaj.ca. 